\title{
Variable-Coefficient Exact Solutions for Nonlinear Differential Equations by a New Bernoulli Equation-Based Subequation Method
}

\author{
Chunxia Qi and Shunliang Huang \\ School of Business, Shandong University of Technology, Zibo, Shandong 255049, China \\ Correspondence should be addressed to Chunxia Qi; qichunxiasdut@126.com
}

Received 20 January 2013; Revised 10 March 2013; Accepted 11 March 2013

Academic Editor: Ebrahim Momoniat

Copyright (C) 2013 C. Qi and S. Huang. This is an open access article distributed under the Creative Commons Attribution License, which permits unrestricted use, distribution, and reproduction in any medium, provided the original work is properly cited.

A new Bernoulli equation-based subequation method is proposed to establish variable-coefficient exact solutions for nonlinear differential equations. For illustrating the validity of this method, we apply it to the asymmetric $(2+1)$-dimensional NNV system and the Kaup-Kupershmidt equation. As a result, some new exact solutions with variable functions coefficients for them are successfully obtained.

\section{Introduction}

Nonlinear differential equations (NLDEs) can be used to describe many nonlinear phenomena such as fluid mechanics, plasma physics, optical fibers, biology, solid state physics, chemical kinematics, and chemical physics. Recently, research for seeking exact analytical solutions of NLDEs has been a hot topic, and many powerful and efficient methods to find exact solutions have been presented so far. For example, these methods include the known homogeneous balance method [1,2], the tanh method [3-5], the inverse scattering transform [6], the Backlund transform [7, 8], the Hirotas bilinear method $[9,10]$, the generalized Riccati subequation method $[11,12]$, the Jacobi elliptic function expansion $[13,14]$, the $F$-expansion method [15], the exp-function expansion method [16, 17], and the $\left(G^{\prime} / G\right)$-expansion method [18, 19]. However, we notice that most of the existing methods are accompanied with constant coefficients, while very few methods are concerned with variable coefficients.

In this paper, by introducing a new ansatz, we develop a new Bernoulli equation-based sub equation method for obtaining variable-coefficient exact solutions for NLDEs. First we give the description of the Bernoulli equation-based subequation method. Then we apply the method to solve the asymmetric (2+1)-dimensional NNV system and the KaupKupershmidt equation. Some conclusions are presented at the end of the paper.

\section{Description of the Bernoulli Equation-Based Subequation Method}

We consider the following Bernoulli equation:

$$
G^{\prime}+\lambda G=G^{2}
$$

where $\lambda \neq 0$ is a complex number and $G=G(\xi)$. The solutions of (1) are denoted by

$$
G(\xi)=\frac{\lambda}{1+\lambda d e^{\lambda \xi}}
$$

where $d$ is an arbitrary constant. In particular, when $\lambda$ is a real number and $d=1 / \lambda$, we obtain

$$
G(\xi)=\frac{\lambda}{2}\left(1-\tanh \left(\frac{\lambda \xi}{2}\right)\right) .
$$


When $d=1 / \lambda, \lambda=i \widetilde{\lambda}$, where $\tilde{\lambda}$ is a real number and $i$ is the unit of imaginary number, we obtain

$$
G(\xi)=\frac{\lambda}{2}-\frac{\lambda i}{2} \tan \left(\frac{\tilde{\lambda} \xi}{2}\right) .
$$

Suppose that a nonlinear equation, say in two or three independent variables $x, y, t$, is given by

$$
P\left(u, u_{t}, u_{x}, u_{y}, u_{t t}, u_{x t}, u_{x x}, u_{x y}, \ldots\right)=0,
$$

where $u=u(x, y, t)$ is an unknown function and $P$ is a polynomial in $u=u(x, y, t)$ and its various partial derivatives, in which the highest order derivatives and nonlinear terms are involved.

Step 1. Suppose that

$$
u(x, y, t)=u(\xi), \quad \xi=\xi(x, y, t),
$$

and then (5) can be turned into the following form:

$$
\widetilde{P}\left(u, u^{\prime}, u^{\prime \prime}, \ldots\right)=0 .
$$

Step 2. Suppose that the solution of (7) can be expressed by a polynomial in $G$ as follows:

$$
\begin{aligned}
u(\xi)= & a_{m}(x, y, t) G^{m}+a_{m-1}(x, y, t) G^{m-1} \\
& +\cdots+a_{0}(x, y, t),
\end{aligned}
$$

where $G=G(\xi)$ satisfies $(1)$ and $a_{m}(x, y, t), a_{m-1}(x, y, t), \ldots$, $a_{0}(x, y, t)$ are all unknown functions to be determined later with $a_{m}(x, y, t) \neq 0$. The positive integer $m$ can be determined by considering the homogeneous balance between the highest order derivatives and nonlinear terms appearing in (7).

Step 3. Substituting (8) into (7) and using (1), collecting all terms with the same order of $G$ together, the lefthand side of (7) is converted to another polynomial in G. Equating each coefficient of this polynomial to zero yields a set of partial differential equations for $a_{m}(x, y, t), a_{m-1}(x, y, t), \ldots, \xi(x, y, t), \lambda$.

Step 4. Solving the equations system in Step 3, and using the solutions of (1), we can construct exact coefficient function solutions of (7).

Remark 1. As the partial differential equations in Step 3 are usually overdetermined, we may choose some special forms of $a_{m}, a_{m-1}, \ldots, a_{0}$ as done in the following.

\section{Application of the Bernoulli Equation-Based Subequation Method to Some NLDEs}

3.1. Asymmetric (2+1)-Dimensional NNV System. We consider the $(2+1)$-dimensional asymmetric NNV system [2022]:

$$
\begin{gathered}
u_{t}-u_{x x x}+\alpha(u v)_{x}=0, \\
u_{x}+\beta v_{y}=0,
\end{gathered}
$$

where $\alpha$ and $\beta$ are arbitrary nonzero constants.
Assume that $u(x, y, t)=U(\xi)$, where $\xi=\xi(x, y, t)$, and then (9) can be turned into

$$
\begin{gathered}
\xi_{t}^{\prime} U^{\prime}-\left(\xi_{x}^{3} U^{\prime \prime \prime}+3 \xi_{x} \xi_{x x} U^{\prime \prime}+\xi_{x x x} U^{\prime}\right)+\alpha \xi_{x}(U V)^{\prime}=0 \\
\xi_{x}^{\prime} U^{\prime}+\beta \xi_{y} V^{\prime}=0 .
\end{gathered}
$$

Suppose that the solutions of (10)-(11) can be expressed by a polynomial in $G$ as follows:

$$
U(\xi)=\sum_{i=0}^{m} a_{i}(y, t) G^{i}, \quad V(\xi)=\sum_{i=0}^{n} b_{i}(y, t) G^{i}
$$

where $a_{i}(y, t), b_{i}(y, t)$ are underdetermined functions and $G=G(\xi)$ satisfies (1). Balancing the order of $U^{\prime \prime \prime}$ and $(U V)^{\prime}$ in (10) and the order of $U^{\prime}$ and $V^{\prime}$ in (11), we can obtain $m+3=m+n+1, m+1=n+1 \Rightarrow m=2, n=2$. So we have

$$
\begin{gathered}
U(\xi)=a_{2}(y, t) G^{2}+a_{1}(y, t) G+a_{0}(y, t), \\
V(\xi)=b_{2}(y, t) G^{2}+b_{1}(y, t) G+b_{0}(y, t) .
\end{gathered}
$$

Substituting (13) into (10)-(11) and collecting all the terms with the same power of $G$ together, equating each coefficient to zero yields a set of underdetermined partial differential equations for $a_{i}(x, y, t), b_{i}(x, y, t), i=0,1,2$, and $\xi(x, y, t)$. Solving these equations yields the following.

Case 1. Consider

$$
\begin{gathered}
\xi(x, y, t)=C_{1} x-\int C_{1} \alpha F_{1}(t) d t+C_{1}^{3} \lambda^{2} t+F_{2}(y), \\
a_{2}(y, t)=-\frac{6 \beta C_{1} F_{2}^{\prime}(y)}{\alpha}, \quad a_{1}(y, t)=\frac{6 \beta \lambda C_{1} F_{2}^{\prime}(y)}{\alpha}, \\
a_{0}(y, t)=0, \quad b_{2}(y, t)=\frac{6 C_{1}^{2}}{\alpha}, \\
b_{1}(y, t)=-\frac{6 C_{1}^{2} \lambda}{\alpha}, \quad b_{0}(y, t)=F_{1}(t),
\end{gathered}
$$

where $C_{1}$ is an arbitrary constant and $F_{1}(t)$ and $(y)$ are two arbitrary functions with respect to the variables $t$ and $y$, respectively.

\section{Case 2. Consider}

$$
\begin{aligned}
& \xi(x, y, t) \\
& \quad=C_{1} x+\int \frac{-\alpha \beta C_{1} C_{2} F_{1}(t)+C_{1}^{2}\left(\alpha+C_{1} C_{2} \beta \lambda^{2}\right)}{\beta C_{2}} d t
\end{aligned}
$$




$$
\begin{array}{cc}
+C_{2} \int F_{2}(y) d y+C_{3}, \\
a_{2}(y, t)=-\frac{6 \beta C_{1} C_{2} F_{2}(y)}{\alpha}, & a_{1}(y, t)=\frac{6 \beta \lambda C_{1} C_{2} F_{2}(y)}{\alpha}, \\
a_{0}(y, t)=F_{2}(y), & b_{2}(y, t)=\frac{6 C_{1}^{2}}{\alpha}, \\
b_{1}(y, t)=-\frac{6 C_{1}^{2} \lambda}{\alpha}, & b_{0}(y, t)=F_{1}(t),
\end{array}
$$

where $C_{1}, C_{2}$, and $C_{3}$ are arbitrary constants and $F_{1}(t)$ and $F_{2}(y)$ are two arbitrary functions with respect to the variables $t$ and $y$, respectively.

Case 3. Consider

$$
\begin{aligned}
& \xi(x, y, t)=-\frac{\alpha F_{1}(y)+\sqrt{\left(\alpha^{2}+4 \alpha \beta^{2} \lambda^{2} C_{1} C_{2}^{2}\right) F_{1}^{2}(y)}}{2 \beta C_{2} \lambda^{2} F_{1}(y)} x \\
& +C_{2} \int F_{1}(y) d y+C_{3}, \\
& a_{0}(y, t)=F_{1}(y), \quad b_{0}(y, t)=C_{1}, \\
& a_{2}(y, t)=\frac{3\left(\alpha F_{1}(y)+\sqrt{\left(\alpha^{2}+4 \alpha \beta^{2} \lambda^{2} C_{1} C_{2}^{2}\right) F_{1}^{2}(y)}\right)}{\lambda^{2} \alpha}, \\
& a_{1}(y, t)=-\frac{3\left(\alpha F_{1}(y)+\sqrt{\left(\alpha^{2}+4 \alpha \beta^{2} \lambda^{2} C_{1} C_{2}^{2}\right) F_{1}^{2}(y)}\right)}{\lambda \alpha}, \\
& b_{2}(y, t)=\frac{3\left[\alpha F_{1}(y)+\sqrt{\left(\alpha^{2}+4 \alpha \beta^{2} \lambda^{2} C_{1} C_{2}^{2}\right) F_{1}^{2}(y)}\right]^{2}}{2 \alpha \beta^{2} \lambda^{4} C_{2}^{2} F_{1}^{2}(y)}, \\
& b_{1}(y, t)=-\frac{3\left[\alpha F_{1}(y)+\sqrt{\left(\alpha^{2}+4 \alpha \beta^{2} \lambda^{2} C_{1} C_{2}^{2}\right) F_{1}^{2}(y)}\right]^{2}}{2 \alpha \beta^{2} \lambda^{3} C_{2}^{2} F_{1}^{2}(y)}
\end{aligned}
$$

where $C_{1}, C_{2}$, and $C_{3}$ are arbitrary constants and $F_{1}(y)$ is an arbitrary function.

Case 4. Consider

$$
\begin{gathered}
\xi(x, y, t)=-\frac{\sqrt{C_{1} \alpha} x}{\lambda}+F_{1}(y), \\
a_{2}(y, t)=\frac{6 \beta C_{1} F_{1}^{\prime}(y)}{\lambda \sqrt{C_{1} \alpha}}, \\
a_{1}(y, t)=-\frac{6 \beta C_{1} F_{1}^{\prime}(y)}{\sqrt{C_{1} \alpha}}, \quad a_{0}(y, t)=0, \\
b_{2}(y, t)=\frac{6 C_{1}}{\lambda^{2}}, \quad b_{1}(y, t)=-\frac{6 C_{1}}{\lambda}, \quad b_{0}(y, t)=C_{1},
\end{gathered}
$$

where $C_{1}$ is an arbitrary constant with $C_{1} \alpha>0$ and $F_{1}(y)$ is an arbitrary function.

Substituting the results in the four cases mentioned previously into (13), and combining with the solutions of (1) as denoted in (2), we can obtain a rich variety of exact solutions to the asymmetric (2+1)-dimensional NNV system as follows.

Family 1. One has

$$
\begin{aligned}
u_{1}(x, y, t)= & -\frac{6 \beta C_{1} F_{2}^{\prime}(y)}{\alpha} \\
& \times\left(\frac{\lambda}{1+\lambda d e^{\lambda\left[C_{1} x-\int C_{1} \alpha F_{1}(t) d t+C_{1}^{3} \lambda^{2} t+F_{2}(y)\right]}}\right)^{2} \\
& +\frac{6 \beta \lambda C_{1} F_{2}^{\prime}(y)}{\alpha} \\
& \times\left(\frac{\lambda}{1+\lambda d e^{\lambda\left[C_{1} x-\int C_{1} \alpha F_{1}(t) d t+C_{1}^{3} \lambda^{2} t+F_{2}(y)\right]}}\right), \\
v_{1}(x, y, t) & \frac{6 C_{1}^{2}}{\alpha}\left(\frac{\lambda}{1+\lambda d e^{\lambda\left[C_{1} x-\int C_{1} \alpha F_{1}(t) d t+C_{1}^{3} \lambda^{2} t+F_{2}(y)\right]}}\right)^{2} \\
& -\frac{6 C_{1}^{2} \lambda}{\alpha}\left(\frac{\lambda}{1+\lambda d e^{\lambda\left[C_{1} x-\int C_{1} \alpha F_{1}(t) d t+C_{1}^{3} \lambda^{2} t+F_{2}(y)\right]}}\right) \\
& +F_{1}(t) .
\end{aligned}
$$

Family 2. One has

$$
\begin{aligned}
u_{2}(x, y, t)= & -\frac{6 \beta C_{1} C_{2} F_{2}(y)}{\alpha}\left(\frac{\lambda}{1+\lambda d e^{\lambda \xi}}\right)^{2} \\
& +\frac{6 \beta \lambda C_{1} C_{2} F_{2}(y)}{\alpha}\left(\frac{\lambda}{1+\lambda d e^{\lambda \xi}}\right)+F_{2}(y), \\
v_{2}(x, y, t)= & \frac{6 C_{1}^{2}}{\alpha}\left(\frac{\lambda}{1+\lambda d e^{\lambda \xi}}\right)^{2} \\
& -\frac{6 C_{1}^{2} \lambda}{\alpha}\left(\frac{\lambda}{1+\lambda d e^{\lambda \xi}}\right)+F_{1}(t),
\end{aligned}
$$

where $\xi=C_{1} x+\int\left(\left(-\alpha \beta C_{1} C_{2} F_{1}(t)+C_{1}^{2}\left(\alpha+C_{1} C_{2} \beta \lambda^{2}\right)\right) /\right.$ $\left.\beta C_{2}\right) d t+C_{2} \int F_{2}(y) d y+C_{3}$.

Family 3. One has

$$
\begin{aligned}
u_{3}(x, y, t) & \\
= & \frac{3\left(\alpha F_{1}(y)+\sqrt{\left(\alpha^{2}+4 \alpha \beta^{2} \lambda^{2} C_{1} C_{2}^{2}\right) F_{1}^{2}(y)}\right)}{\lambda^{2} \alpha} \\
& \times\left(\frac{\lambda}{1+\lambda d e^{\lambda \xi}}\right)^{2}
\end{aligned}
$$




$$
\begin{aligned}
& -\frac{3\left(\alpha F_{1}(y)+\sqrt{\left(\alpha^{2}+4 \alpha \beta^{2} \lambda^{2} C_{1} C_{2}^{2}\right) F_{1}^{2}(y)}\right)}{\lambda \alpha} \\
& \times\left(\frac{\lambda}{1+\lambda d e^{\lambda \xi}}\right)+F_{1}(y),
\end{aligned}
$$

$$
v_{3}(x, y, t)
$$

$$
\begin{aligned}
= & \frac{3\left[\alpha F_{1}(y)+\sqrt{\left(\alpha^{2}+4 \alpha \beta^{2} \lambda^{2} C_{1} C_{2}^{2}\right) F_{1}^{2}(y)}\right]^{2}}{2 \alpha \beta^{2} \lambda^{4} C_{2}^{2} F_{1}^{2}(y)} \\
& \times\left(\frac{\lambda}{1+\lambda d e^{\lambda \xi}}\right)^{2}
\end{aligned}
$$$$
-\frac{3\left[\alpha F_{1}(y)+\sqrt{\left(\alpha^{2}+4 \alpha \beta^{2} \lambda^{2} C_{1} C_{2}^{2}\right) F_{1}^{2}(y)}\right]^{2}}{2 \alpha \beta^{2} \lambda^{3} C_{2}^{2} F_{1}^{2}(y)}
$$$$
\times\left(\frac{\lambda}{1+\lambda d e^{\lambda \xi}}\right)+C_{1},
$$

where $\xi=-\left(\left(\alpha F_{1}(y)+\sqrt{\left(\alpha^{2}+4 \alpha \beta^{2} \lambda^{2} C_{1} C_{2}^{2}\right) F_{1}^{2}(y)}\right) /\right.$ $\left.2 \beta C_{2} \lambda^{2} F_{1}(y)\right) x+C_{2} \int F_{1}(y) d y+C_{3}$.

Family 4. Consider

$$
\begin{gathered}
u_{4}(x, y, t)=\frac{6 \beta C_{1} F_{1}^{\prime}(y)}{\lambda \sqrt{C_{1} \alpha}}\left(\frac{\lambda}{1+\lambda d e^{\lambda \xi}}\right)^{2} \\
-\frac{6 \beta C_{1} F_{1}^{\prime}(y)}{\sqrt{C_{1} \alpha}}\left(\frac{\lambda}{1+\lambda d e^{\lambda \xi}}\right), \\
v_{4}(x, y, t)=\frac{6 C_{1}}{\lambda^{2}}\left(\frac{\lambda}{1+\lambda d e^{\lambda \xi}}\right)^{2}-\frac{6 C_{1}}{\lambda}\left(\frac{\lambda}{1+\lambda d e^{\lambda \xi}}\right)+C_{1},
\end{gathered}
$$

where $\xi=-\left(\sqrt{C_{1} \alpha} x / \lambda\right)+F_{1}(y)$.

Particularly, by a combination between Cases 1-4 and (3) we can obtain some special hyperbolic function solutions as follows:

$$
\begin{aligned}
u_{5}(x, y, t)= & -\frac{6 \beta C_{1} F_{2}^{\prime}(y)}{\alpha}\left[\frac{\lambda}{2}\left(1-\tanh \left(\frac{\lambda \xi}{2}\right)\right)\right]^{2} \\
& +\frac{6 \beta \lambda C_{1} F_{2}^{\prime}(y)}{\alpha}\left[\frac{\lambda}{2}\left(1-\tanh \left(\frac{\lambda \xi}{2}\right)\right)\right], \\
v_{5}(x, y, t)= & \frac{6 C_{1}^{2}}{\alpha}\left[\frac{\lambda}{2}\left(1-\tanh \left(\frac{\lambda \xi}{2}\right)\right)\right]^{2} \\
& -\frac{6 C_{1}^{2} \lambda}{\alpha}\left[\frac{\lambda}{2}\left(1-\tanh \left(\frac{\lambda \xi}{2}\right)\right)\right]+F_{1}(t),
\end{aligned}
$$

where $\xi=C_{1} x-\int C_{1} \alpha F_{1}(t) d t+C_{1}^{3} \lambda^{2} t+F_{2}(y)$.

\section{Consider}

$$
\begin{aligned}
u_{6}(x, y, t)= & -\frac{6 \beta C_{1} C_{2} F_{2}(y)}{\alpha}\left[\frac{\lambda}{2}\left(1-\tanh \left(\frac{\lambda \xi}{2}\right)\right)\right]^{2} \\
& +\frac{6 \beta \lambda C_{1} C_{2} F_{2}(y)}{\alpha}\left[\frac{\lambda}{2}\left(1-\tanh \left(\frac{\lambda \xi}{2}\right)\right)\right] \\
& +F_{2}(y), \\
v_{6}(x, y, t)= & \frac{6 C_{1}^{2}}{\alpha}\left[\frac{\lambda}{2}\left(1-\tanh \left(\frac{\lambda \xi}{2}\right)\right)\right]^{2} \\
& -\frac{6 C_{1}^{2} \lambda}{\alpha}\left[\frac{\lambda}{2}\left(1-\tanh \left(\frac{\lambda \xi}{2}\right)\right)\right]+F_{1}(t),
\end{aligned}
$$

where $\xi=C_{1} x+\int\left(\left(-\alpha \beta C_{1} C_{2} F_{1}(t)+C_{1}^{2}\left(\alpha+C_{1} C_{2} \beta \lambda^{2}\right)\right) /\right.$ $\left.\beta C_{2}\right) d t+C_{2} \int F_{2}(y) d y+C_{3}$.

\section{Consider}

$$
\begin{aligned}
u_{7}(x, y, t)= & \frac{3\left(\alpha F_{1}(y)+\sqrt{\left(\alpha^{2}+4 \alpha \beta^{2} \lambda^{2} C_{1} C_{2}^{2}\right) F_{1}^{2}(y)}\right)}{\lambda^{2} \alpha} \\
& \times\left[\frac{\lambda}{2}\left(1-\tanh \left(\frac{\lambda \xi}{2}\right)\right)\right]^{2} \\
& -\frac{3\left(\alpha F_{1}(y)+\sqrt{\left(\alpha^{2}+4 \alpha \beta^{2} \lambda^{2} C_{1} C_{2}^{2}\right) F_{1}^{2}(y)}\right)}{\lambda \alpha} \\
& \times\left[\frac{\lambda}{2}\left(1-\tanh \left(\frac{\lambda \xi}{2}\right)\right)\right]+F_{1}(y), \\
v_{7}(x, y, t)= & \frac{3\left[\alpha F_{1}(y)+\sqrt{\left(\alpha^{2}+4 \alpha \beta^{2} \lambda^{2} C_{1} C_{2}^{2}\right) F_{1}^{2}(y)}\right]^{2}}{2 \alpha \beta^{2} \lambda^{4} C_{2}^{2} F_{1}^{2}(y)} \\
& \times\left[\frac{\lambda}{2}\left(1-\tanh \left(\frac{\lambda \xi}{2}\right)\right)\right]^{2} \\
& -\frac{3\left[\alpha F_{1}(y)+\sqrt{\left(\alpha^{2}+4 \alpha \beta^{2} \lambda^{2} C_{1} C_{2}^{2}\right) F_{1}^{2}(y)}\right]^{2}}{2 \alpha \beta^{2} \lambda^{3} C_{2}^{2} F_{1}^{2}(y)} \\
& \times\left[\frac{\lambda}{2}\left(1-\tanh \left(\frac{\lambda \xi}{2}\right)\right)\right]+C_{1},
\end{aligned}
$$

where $\xi=-\left(\left(\alpha F_{1}(y)+\sqrt{\left.\left(\alpha^{2}+4 \alpha \beta^{2} \lambda^{2} C_{1} C_{2}^{2}\right) F_{1}^{2}(y)\right)}\right) /\right.$ $\left.2 \beta C_{2} \lambda^{2} F_{1}(y)\right) x+C_{2} \int F_{1}(y) d y+C_{3}$. 
Consider

$$
\begin{aligned}
u_{8}(x, y, t)= & \frac{6 \beta C_{1} F_{1}^{\prime}(y)}{\lambda \sqrt{C_{1} \alpha}}\left[\frac{\lambda}{2}\left(1-\tanh \left(\frac{\lambda \xi}{2}\right)\right)\right]^{2} \\
& -\frac{6 \beta C_{1} F_{1}^{\prime}(y)}{\sqrt{C_{1} \alpha}}\left[\frac{\lambda}{2}\left(1-\tanh \left(\frac{\lambda \xi}{2}\right)\right)\right], \\
v_{8}(x, y, t)= & \frac{6 C_{1}}{\lambda^{2}}\left[\frac{\lambda}{2}\left(1-\tanh \left(\frac{\lambda \xi}{2}\right)\right)\right]^{2} \\
& -\frac{6 C_{1}}{\lambda}\left[\frac{\lambda}{2}\left(1-\tanh \left(\frac{\lambda \xi}{2}\right)\right)\right]+C_{1},
\end{aligned}
$$

where $\xi=\left(-\sqrt{C_{1} \alpha} x / \lambda\right)+F_{1}(y)$.

By a combination with (4) we can obtain some special trigonometric function solutions as follows:

$$
\begin{aligned}
u_{9}(x, y, t)= & -\frac{6 \beta C_{1} F_{2}^{\prime}(y)}{\alpha}\left[\frac{\lambda}{2}-\frac{\lambda i}{2} \tan \left(\frac{\tilde{\lambda} \xi}{2}\right)\right]^{2} \\
& +\frac{6 \beta \lambda C_{1} F_{2}^{\prime}(y)}{\alpha}\left[\frac{\lambda}{2}-\frac{\lambda i}{2} \tan \left(\frac{\tilde{\lambda} \xi}{2}\right)\right], \\
v_{9}(x, y, t)= & \frac{6 C_{1}^{2}}{\alpha}\left[\frac{\lambda}{2}-\frac{\lambda i}{2} \tan \left(\frac{\tilde{\lambda} \xi}{2}\right)\right]^{2} \\
& -\frac{6 C_{1}^{2} \lambda}{\alpha}\left[\frac{\lambda}{2}-\frac{\lambda i}{2} \tan \left(\frac{\tilde{\lambda} \xi}{2}\right)\right]+F_{1}(t),
\end{aligned}
$$

where $\xi=C_{1} x-\int C_{1} \alpha F_{1}(t) d t+C_{1}^{3} \lambda^{2} t+F_{2}(y), \tilde{\lambda}$ is a real number and $\lambda=i \widetilde{\lambda}$.

One has

$$
\begin{aligned}
u_{10}(x, y, t)= & -\frac{6 \beta C_{1} C_{2} F_{2}(y)}{\alpha}\left[\frac{\lambda}{2}-\frac{\lambda i}{2} \tan \left(\frac{\tilde{\lambda} \xi}{2}\right)\right]^{2} \\
& +\frac{6 \beta \lambda C_{1} C_{2} F_{2}(y)}{\alpha}\left[\frac{\lambda}{2}-\frac{\lambda i}{2} \tan \left(\frac{\tilde{\lambda} \xi}{2}\right)\right] \\
& +F_{2}(y), \\
v_{10}(x, y, t)= & \frac{6 C_{1}^{2}}{\alpha}\left[\frac{\lambda}{2}-\frac{\lambda i}{2} \tan \left(\frac{\tilde{\lambda} \xi}{2}\right)\right]^{2} \\
& -\frac{6 C_{1}^{2} \lambda}{\alpha}\left[\frac{\lambda}{2}-\frac{\lambda i}{2} \tan \left(\frac{\tilde{\lambda} \xi}{2}\right)\right]+F_{1}(t),
\end{aligned}
$$

where $\xi=C_{1} x+\int\left(\left(-\alpha \beta C_{1} C_{2} F_{1}(t)+C_{1}^{2}\left(\alpha+C_{1} C_{2} \beta \lambda^{2}\right)\right) /\right.$ $\left.\beta C_{2}\right) d t+C_{2} \int F_{2}(y) d y+C_{3}$.
One has

$$
\begin{aligned}
u_{11}(x, y, t)= & \frac{3\left(\alpha F_{1}(y)+\sqrt{\left(\alpha^{2}+4 \alpha \beta^{2} \lambda^{2} C_{1} C_{2}^{2}\right) F_{1}^{2}(y)}\right)}{\lambda^{2} \alpha} \\
& \times\left[\frac{\lambda}{2}-\frac{\lambda i}{2} \tan \left(\frac{\tilde{\lambda} \xi}{2}\right)\right]^{2} \\
& -\frac{3\left(\alpha F_{1}(y)+\sqrt{\left(\alpha^{2}+4 \alpha \beta^{2} \lambda^{2} C_{1} C_{2}^{2}\right) F_{1}^{2}(y)}\right)}{\lambda \alpha} \\
& \times\left[\frac{\lambda}{2}-\frac{\lambda i}{2} \tan \left(\frac{\tilde{\lambda} \xi}{2}\right)\right]+F_{1}(y), \\
v_{11}(x, y, t)= & \frac{\left.3 F_{1}(y)+\sqrt{\left(\alpha^{2}+4 \alpha \beta^{2} \lambda^{2} C_{1} C_{2}^{2}\right) F_{1}^{2}(y)}\right]^{2}}{2 \alpha \beta^{2} \lambda^{4} C_{2}^{2} F_{1}^{2}(y)} \\
& \times\left[\frac{\lambda}{2}-\frac{\lambda i}{2} \tan \left(\frac{\lambda \xi}{2}\right)\right]^{2} \\
& -\frac{3\left[\alpha F_{1}(y)+\sqrt{\left(\alpha^{2}+4 \alpha \beta^{2} \lambda^{2} C_{1} C_{2}^{2}\right) F_{1}^{2}(y)}\right]^{2}}{2 \alpha \beta^{2} \lambda^{3} C_{2}^{2} F_{1}^{2}(y)} \\
& \left.\left.\times \frac{\tilde{\lambda} \xi}{2}\right)\right]+C_{1},
\end{aligned}
$$

where $\xi=-\left(\left(\alpha F_{1}(y)+\sqrt{\left(\alpha^{2}+4 \alpha \beta^{2} \lambda^{2} C_{1} C_{2}^{2}\right) F_{1}^{2}(y)}\right) /\right.$ $\left.2 \beta C_{2} \lambda^{2} F_{1}(y)\right) x+C_{2} \int F_{1}(y) d y+C_{3}$.

$$
\begin{aligned}
u_{12}(x, y, t)= & \frac{6 \beta C_{1} F_{1}^{\prime}(y)}{\lambda \sqrt{C_{1} \alpha}}\left[\frac{\lambda}{2}-\frac{\lambda i}{2} \tan \left(\frac{\tilde{\lambda} \xi}{2}\right)\right]^{2} \\
& -\frac{6 \beta C_{1} F_{1}^{\prime}(y)}{\sqrt{C_{1} \alpha}}\left[\frac{\lambda}{2}-\frac{\lambda i}{2} \tan \left(\frac{\tilde{\lambda} \xi}{2}\right)\right], \\
v_{12}(x, y, t)= & \frac{6 C_{1}}{\lambda^{2}}\left[\frac{\lambda}{2}-\frac{\lambda i}{2} \tan \left(\frac{\tilde{\lambda} \xi}{2}\right)\right]^{2} \\
& -\frac{6 C_{1}}{\lambda}\left[\frac{\lambda}{2}-\frac{\lambda i}{2} \tan \left(\frac{\tilde{\lambda} \xi}{2}\right)\right]+C_{1},
\end{aligned}
$$

where $\xi=-\left(\sqrt{C_{1} \alpha} x / \lambda\right)+F_{1}(y)$.

Remark 2. In [20-22], some exact solutions for the asymmetric (2+1)-dimensional NNV system are established using different methods. We note that the established solutions mentioned previously are different from them essentially as they are new exact solutions with variable functions coefficients and have been reported by other authors in the literature. 
3.2. Kaup-Kupershmidt Equation. We consider the following Kaup-Kupershmidt equation [23-25]:

$$
u_{x x x x x}+u_{t}+45 u_{x} u^{2}-\frac{75}{2} u_{x} u_{x x}-15 u u_{x x x}=0
$$

Suppose that $u(x, t)=U(\xi), \xi=\xi(x, t)$, and, furthermore, by balancing the order in (31), we can suppose that

$$
U(\xi)=a_{2}(x, t) G^{2}+a_{1}(x, t) G+a_{0}(x, t),
$$

where $G=G(\xi)$ satisfies (1). Similar to the process mentioned before, we obtain

$$
\begin{gathered}
\xi(x, t)=\frac{2}{\lambda i} \arctan \left(\frac{C_{1}\left(x+C_{2}\right)}{2 \lambda i}\right), \\
a_{2}(x, t)=\frac{128 C_{1}^{2}}{\left(C_{1}^{2}\left(x+C_{2}\right)^{2}-4 \lambda^{2}\right)^{2}}, \\
a_{1}(x, t)=-\frac{64 C_{1}^{3}\left(x+C_{2}\right)}{\left(C_{1}^{2}\left(x+C_{2}\right)^{2}-4 \lambda^{2}\right)^{2}} \\
a_{0}(x, t)=\frac{8 C_{1}^{4}\left(x+C_{2}\right)^{2}}{\left(C_{1}^{2}\left(x+C_{2}\right)^{2}-4 \lambda^{2}\right)^{2}}+\frac{\lambda}{2}
\end{gathered}
$$

Then we can obtain a kind of exact solutions of (31) which is unrelated to time variable as follows:

$$
\begin{aligned}
u_{1}(x, t) & \\
= & \frac{128 C_{1}^{2}}{\left(C_{1}^{2}\left(x+C_{2}\right)^{2}-4 \lambda^{2}\right)^{2}} \\
& \times\left(\frac{\lambda}{1+\lambda d e^{(2 / i) \arctan \left(C_{1}\left(x+C_{2}\right) / 2 \lambda i\right)}}\right)^{2} \\
& -\frac{64 C_{1}^{3}\left(x+C_{2}\right)}{\left(C_{1}^{2}\left(x+C_{2}\right)^{2}-4 \lambda^{2}\right)^{2}} \\
& \times\left(\frac{\lambda}{1+\lambda d e^{(2 / i) \arctan \left(C_{1}\left(x+C_{2}\right) / 2 \lambda i\right)}}\right) \\
& +\frac{8 C_{1}^{4}\left(x+C_{2}\right)^{2}}{\left(C_{1}^{2}\left(x+C_{2}\right)^{2}-4 \lambda^{2}\right)^{2}}+\frac{\lambda}{2} .
\end{aligned}
$$

In particular, by the combination with (3)-(4) we can obtain hyperbolic function and trigonometric function solutions as follows:

$$
\begin{aligned}
u_{2}(x, t)= & \frac{128 C_{1}^{2}}{\left(C_{1}^{2}\left(x+C_{2}\right)^{2}-4 \lambda^{2}\right)^{2}}\left[\frac{\lambda}{2}\left(1-\tanh \left(\frac{\lambda \xi}{2}\right)\right)\right]^{2} \\
& -\frac{64 C_{1}^{3}\left(x+C_{2}\right)}{\left(C_{1}^{2}\left(x+C_{2}\right)^{2}-4 \lambda^{2}\right)^{2}}\left[\frac{\lambda}{2}\left(1-\tanh \left(\frac{\lambda \xi}{2}\right)\right)\right] \\
& +\frac{8 C_{1}^{4}\left(x+C_{2}\right)^{2}}{\left(C_{1}^{2}\left(x+C_{2}\right)^{2}-4 \lambda^{2}\right)^{2}}+\frac{\lambda}{2}, \\
u_{3}(x, t)= & \frac{128 C_{1}^{2}}{\left(C_{1}^{2}\left(x+C_{2}\right)^{2}-4 \lambda^{2}\right)^{2}}\left[\frac{\lambda}{2}-\frac{\lambda i}{2} \tan \left(\frac{\tilde{\lambda} \xi}{2}\right)\right]^{2} \\
& -\frac{64 C_{1}^{3}\left(x+C_{2}\right)}{\left(C_{1}^{2}\left(x+C_{2}\right)^{2}-4 \lambda^{2}\right)^{2}} \frac{\lambda}{2}\left[\frac{\lambda}{2}-\frac{\lambda i}{2} \tan \left(\frac{\tilde{\lambda} \xi}{2}\right)\right] \\
& +\frac{8 C_{1}^{4}\left(x+C_{2}\right)^{2}}{\left(C_{1}^{2}\left(x+C_{2}\right)^{2}-4 \lambda^{2}\right)^{2}}+\frac{\lambda}{2},
\end{aligned}
$$

where $\xi=(2 / \lambda i) \arctan \left(C_{1}\left(x+C_{2}\right) / 2 \lambda i\right), \tilde{\lambda}$ is a real number and $\lambda=i \tilde{\lambda}$.

Remark 3. The previous established solutions for the KaupKupershmidt equation cannot be obtained by the methods in [23-25] and are new exact solutions to our best knowledge.

\section{Conclusions}

We have proposed a Bernoulli equation-based subequation method for solving nonlinear differential equations and applied it to find exact solutions with variable functions coefficients of the asymmetric (2+1)-dimensional asymmetric NNV system and the Kaup-Kupershmidt equation. As a result, some new exact solutions for them have been successfully found. Finally, we note that the proposed method can be applied to solve other nonlinear evolution equations.

\section{Acknowledgments}

This work is partially supported by Humanity and Social Science Youth Foundation of Ministry of Education of China (11YJCZH070). The authors would like to thank the reviewers very much for their valuable suggestions on this paper.

\section{References}

[1] M. L. Wang, "Solitary wave solutions for variant Boussinesq equations," Physics Letters A, vol. 199, no. 3-4, pp. 169-172, 1995.

[2] E. M. E. Zayed, H. A. Zedan, and K. A. Gepreel, "On the solitary wave solutions for nonlinear Hirota-Satsuma coupled KdV of 
equations," Chaos, Solitons \& Fractals, vol. 22, no. 2, pp. 285303, 2004.

[3] M. A. Abdou, "The extended tanh method and its applications for solving nonlinear physical models," Applied Mathematics and Computation, vol. 190, no. 1, pp. 988-996, 2007.

[4] E. Fan, "Extended tanh-function method and its applications to nonlinear equations," Physics Letters A, vol. 277, no. 4-5, pp. 212-218, 2000.

[5] W. Malfliet, "Solitary wave solutions of nonlinear wave equations," American Journal of Physics, vol. 60, no. 7, pp. 650-654, 1992.

[6] M. J. Ablowitz and P. A. Clarkson, Solitons, Nonlinear Evolution Equations and Inverse Scattering, vol. 149 of London Mathematical Society Lecture Note Series, Cambridge University Press, Cambridge, UK, 1991.

[7] M. R. Miura, Backlund Transformation, Springer, Berlin, Germany, 1978.

[8] C. Rogers and W. F. Shadwick, Bäcklund Transformations and Their Applications, vol. 161 of Mathematics in Science and Engineering, Academic Press, New York, NY, USA, 1982.

[9] R. Hirota, "Exact envelope-soliton solutions of a nonlinear wave equation," Journal of Mathematical Physics, vol. 14, pp. 805-809, 1973.

[10] R. Hirota and J. Satsuma, "Soliton solutions of a coupled Korteweg-de Vries equation," Physics Letters A, vol. 85, no. 8-9, pp. 407-408, 1981.

[11] L. Song and H. Zhang, "New exact solutions for the Konopelchenko-Dubrovsky equation using an extended Riccati equation rational expansion method and symbolic computation," Applied Mathematics and Computation, vol. 187, no. 2, pp. 1373-1388, 2007.

[12] W. Li and H. Zhang, "Generalized multiple Riccati equations rational expansion method with symbolic computation to construct exact complexiton solutions of nonlinear partial differential equations," Applied Mathematics and Computation, vol. 197, no. 1, pp. 288-296, 2008.

[13] S. Liu, Z. Fu, S. Liu, and Q. Zhao, "Jacobi elliptic function expansion method and periodic wave solutions of nonlinear wave equations," Physics Letters A, vol. 289, no. 1-2, pp. 69-74, 2001.

[14] Z. Yan, "Abundant families of Jacobi elliptic function solutions of the $(2+1)$-dimensional integrable Davey-Stewartson-type equation via a new method," Chaos, Solitons \& Fractals, vol. 18, no. 2, pp. 299-309, 2003.

[15] M. Wang and X. Li, "Applications of F-expansion to periodic wave solutions for a new Hamiltonian amplitude equation," Chaos, Solitons \& Fractals, vol. 24, no. 5, pp. 1257-1268, 2005.

[16] J. Biazar and Z. Ayati, "Exp and modified Exp function methods for nonlinear Drinfeld-Sokolov system," Journal of King Saud University-Science, vol. 24, pp. 315-318, 2012.

[17] J.-H. He and X.-H. Wu, "Exp-function method for nonlinear wave equations," Chaos, Solitons \& Fractals, vol. 30, no. 3, pp. 700-708, 2006.

[18] M. Wang, X. Li, and J. Zhang, “The $\left(G^{\prime} / G\right)$-expansion method and travelling wave solutions of nonlinear evolution equations in mathematical physics," Physics Letters A, vol. 372, no. 4, pp. 417-423, 2008.

[19] M. Wang, J. Zhang, and X. Li, "Application of the $\left(G^{\prime} / G\right)$ expansion to travelling wave solutions of the Broer-Kaup and the approximate long water wave equations," Applied Mathematics and Computation, vol. 206, no. 1, pp. 321-326, 2008.
[20] S. Zhang and T. Xia, "Variable-coefficient Jacobi elliptic function expansion method for $(2+1)$-dimensional NizhnikNovikov-Vesselov equations," Applied Mathematics and Computation, vol. 218, no. 4, pp. 1308-1316, 2011.

[21] S. Zhang and T. Xia, "A generalized new auxiliary equation method and its applications to nonlinear partial differential equations," Physics Letters A, vol. 363, no. 5-6, pp. 356-360, 2007.

[22] X.-Y. Jiao, J.-H. Wang, and H.-Q. Zhang, "An extended method for constructing travelling wave solutions to nonlinear partial differential equations," Communications in Theoretical Physics, vol. 44, no. 3, pp. 407-414, 2005.

[23] A. Parker, "On soliton solutions of the Kaup-Kupershmidt equation. I. Direct bilinearisation and solitary wave," Physica D, vol. 137, no. 1-2, pp. 25-33, 2000.

[24] A. Parker, "On soliton solutions of the Kaup-Kupershmidt equation. II. "Anomalous” N-soliton solutions," Physica D, vol. 137, no. 1-2, pp. 34-48, 2000.

[25] Q. Feng and B. Zheng, "Traveling wave solutions for two non-linear equations by $\left(G^{\prime} / G\right)$-expansion method," WSEAS Transactions on Information Science and Applications, vol. 7, no. 3, pp. 401-410, 2010. 


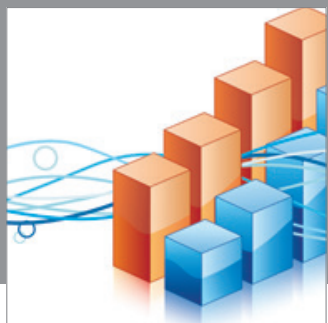

Advances in

Operations Research

mansans

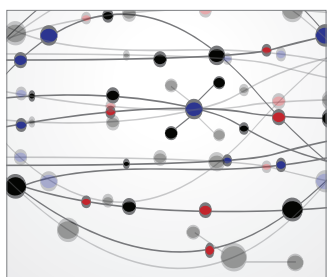

The Scientific World Journal
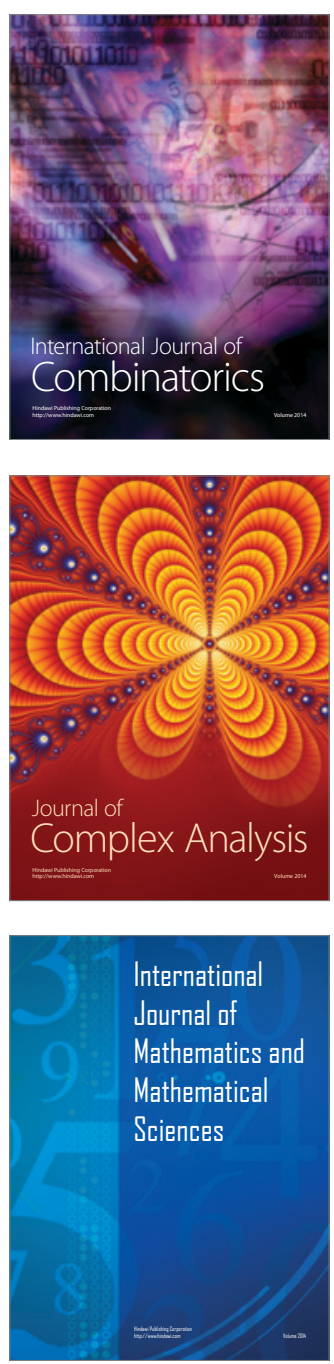
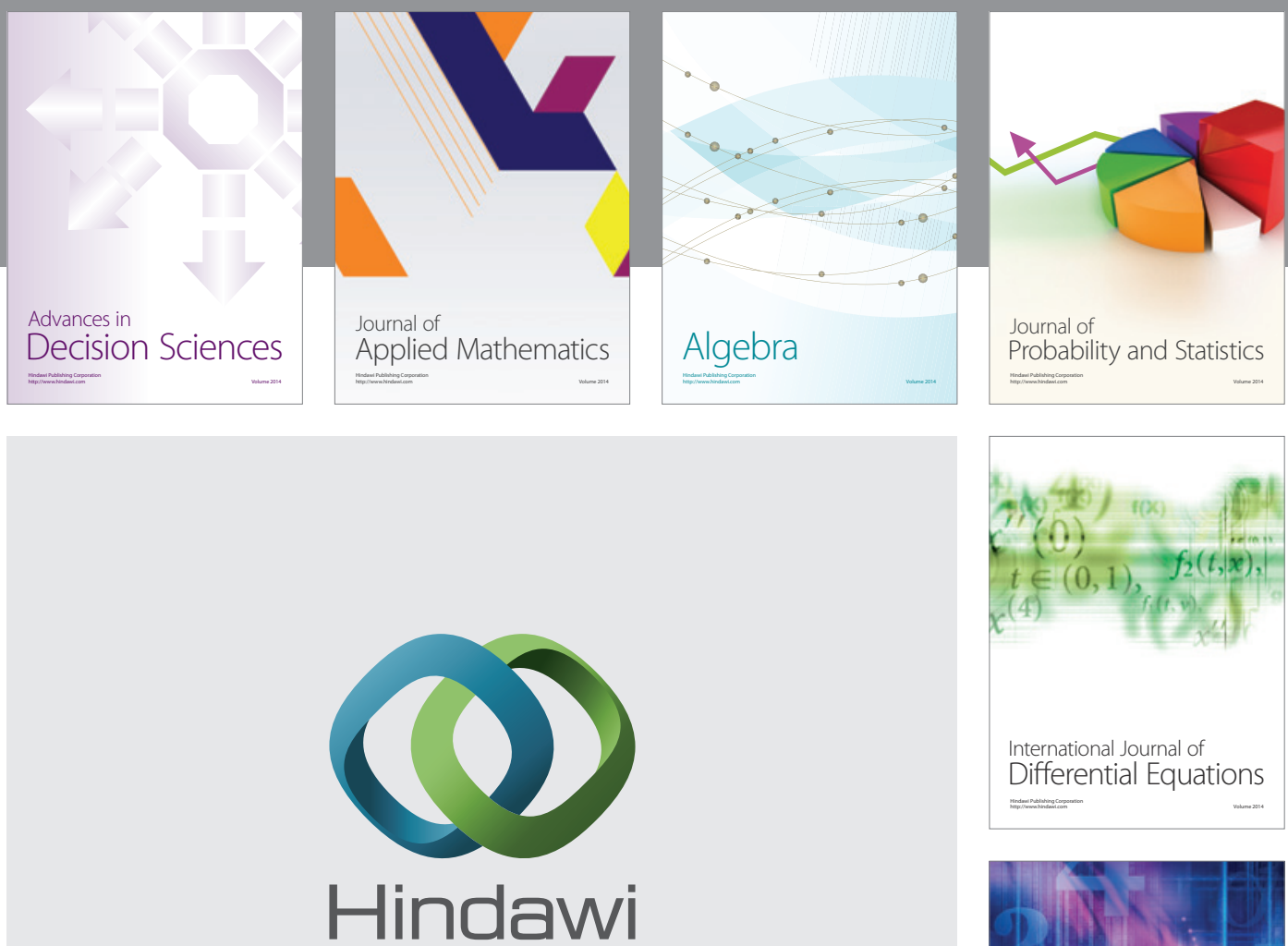

Submit your manuscripts at http://www.hindawi.com
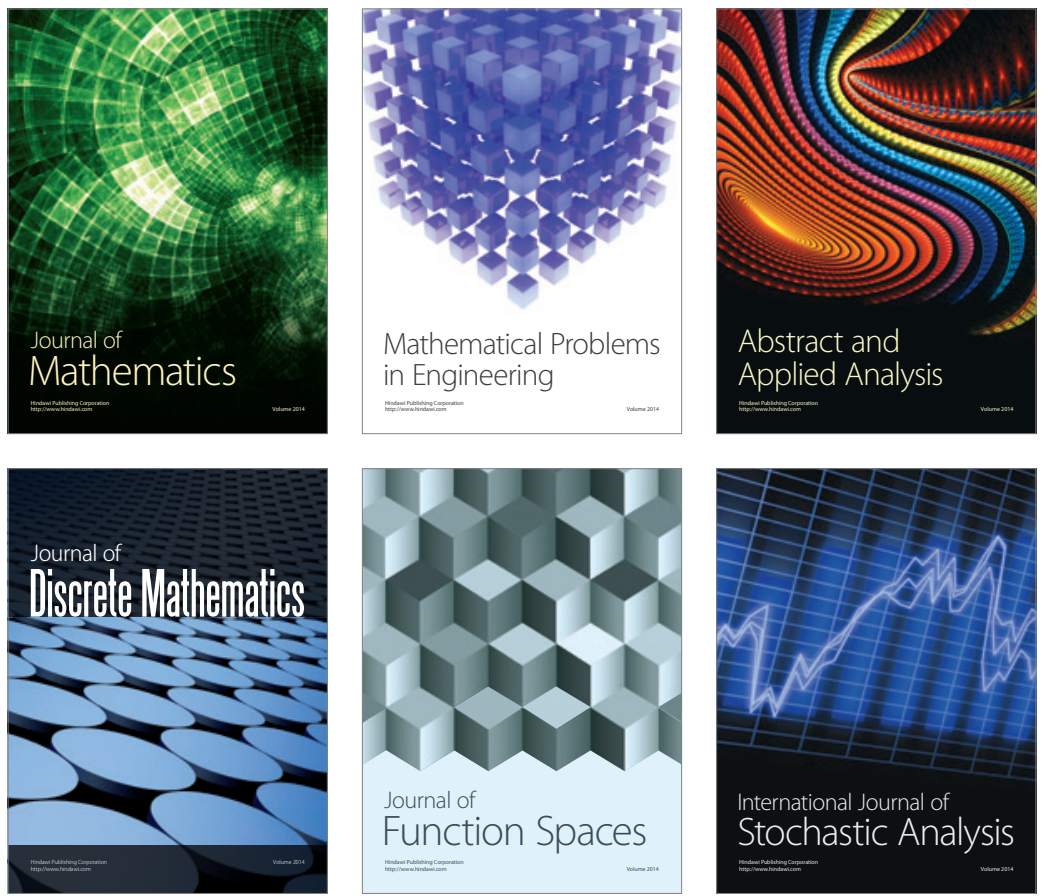

Journal of

Function Spaces

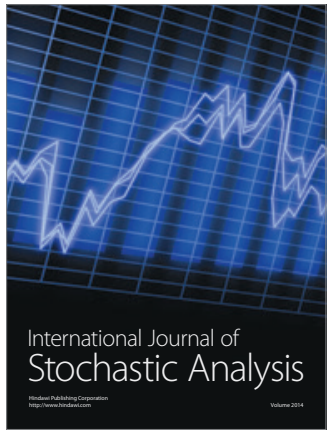

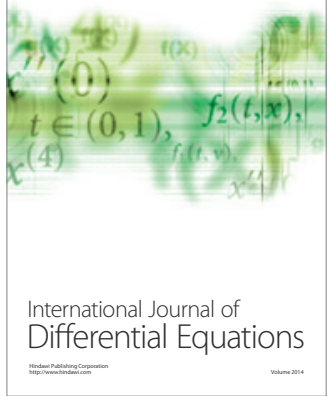
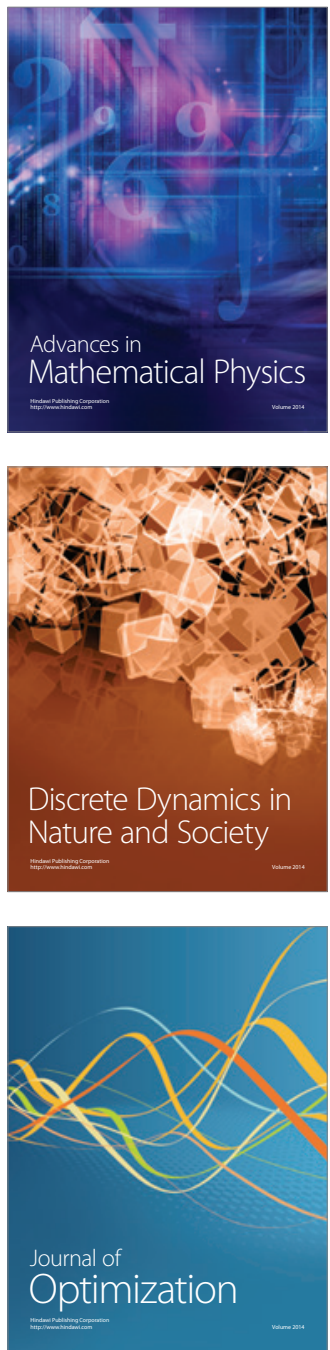